\title{
Penile Cancer by AJCC v6 Stage
}

National Cancer Institute

\section{Source}

National Cancer Institute. Penile Cancer by A/CC v6 Stage. NCI Thesaurus. Code C90520.

A term that refers to the staging of penile carcinoma according to the American Joint Committee on Cancer, 6th edition. 